\title{
25 Research Square

\section{High-performance AlGaN double channel HEMTs with improved drain current density and high breakdown voltage}

Yachao Zhang ( $\sim$ xd_zhangyachao@163.com )

Xidian University

\section{Zhizhe Wang}

China electronic product reliability and environmental tetsting research institute

\section{Shengrui Xu}

Xidian University

Jincheng Zhang

Xidian University

Yue Hao

Xidian University

\section{Nano Express}

Keywords: AIGaN double channel heterostructure, metal organic chemical vapor deposition (MOCVD), high electron mobility transistors

Posted Date: February 20th, 2020

DOI: https://doi.org/10.21203/rs.2.24040/v1

License: (1) This work is licensed under a Creative Commons Attribution 4.0 International License.

Read Full License

Version of Record: A version of this preprint was published at Nanoscale Research Letters on May 20th, 2020. See the published version at https://doi.org/10.1186/s11671-020-03345-6. 


\section{Abstract}

In this work, for the first time, AlGaN double channel heterostructure is proposed and grown by metal organic chemical vapor deposition (MOCVD), and high-performance AIGaN double channel high electron mobility transistors (HEMTs) are fabricated and investigated. The implementation of double channel feature effectively improves the transport properties of AIGaN channel heterostructures. On one hand, the total two dimensional electron gas (2DEG) density is promoted due to the double potential wells along the vertical direction and the enhanced carrier confinement. On the other hand, the average 2DEG density in each channel is reduced, and the mobility is elevated resulted from the suppression of carrier-carrier scattering effect. As a result, the maximum drain current density (Imax) of AIGaN double channel HEMTs reaches $473 \mathrm{~mA} / \mathrm{mm}$ with gate voltage of OV. Moreover, the superior breakdown performance of the AlGaN double channel HEMTs is also demonstrated. These results not only show the great application potential of AIGaN double channel HEMTs in microwave power electronics, but also develops a new thinking for the studies of group III nitride based electronic devices.

\section{Main Text}

Group III nitride based high electron mobility transistors (HEMTs) have be identified as the most promising candidate for next-generation microwave power electronics owing to their fast switching speed and low switching loss [1-5]. Lately, the most advanced nitride HEMTs have achieved initial commercialization up to $650 \mathrm{~V}$. However, with the maturity of device fabrication technology, it has become increasingly difficult to further scaling up the breakdown voltages (BV) and improving the device reliability at high temperatures. Therefore, in view of the larger bandgap and superior thermal stability of AlGaN over GaN, AlGaN channel devices have been proposed as promising candidate to further improve the performance limits of nitride HEMTs in high-voltage and high-temperature applications [6-13]. Recently, the successful implementation of AIGaN channel metal-oxide-semiconductor field-effecttransistors (MOSFETs) have also been reported [14].

However, the limitations of the previously reported AlGaN channel devices are equally obvious. On one hand, on account of the ternary alloy disordered scattering effect, the two dimensional electron gas (2DEG) mobility in AlGaN channel is much lower than that in $\mathrm{GaN}$ channel. As a result, the current drive capacity of AIGaN channel devices are much lower than that of the traditional GaN channel devices. On the other hand, in order to induce the same amount of 2DEG in AlGaN channel, the Al component in AlGaN barrier layer should be higher than that of conventional GaN channel heterostructures, which will increase the difficulties in material growth process. These contradiction seriously inhibit the widespread application of AIGaN channel devices, and the optimization of heterostructure layout are urgently needed.

Double channel (DC) technique is an intriguing approach to promote the channel carrier density of nitride based heterostructures without any adverse impact on the electron mobility, and the current conduction capability of the devices will be improved [15-17]. However, there is few report on the AlGaN double channel heterostructures or electron devices up to now. In this work, for the first time, AlGaN double 
channel heterostructure is proposed and grown to resolve the contradiction between the current drive capability and breakdown performance of nitride based electron device. Further, high performance AIGaN double channel HEMTs based on the novel heterostructures are fabricated and investigated in detail.

The cross section schematic of the AlGaN double channel heterostructure is shown in Fig. 1, and the growth processes can be summarized as follow. Firstly, $1600 \mathrm{~nm}$ GaN buffer layer was grown on the sapphire substrate. Then, $500 \mathrm{~nm}$ graded AIGaN buffer layer with Al composition increasing from 0 to $10 \%$ was grown, which was beneficial to suppress the formation of parasitic channel. Whereafter, $100 \mathrm{~nm}$ lower AIGaN channel, $1 \mathrm{~nm}$ AIN interlayer and $23 \mathrm{~nm}$ lower AIGaN barrier were grown successively, and the Al compositions in the channel and barrier layers are $10 \%$ and $31 \%$, respectively. Finally, $30 \mathrm{~nm}$ upper AlGaN channel, $1 \mathrm{~nm}$ AIN interlayer and $23 \mathrm{~nm}$ upper AlGaN barrier layers were grown, for which the compositions were the same with the lower layers. The conduction band diagram of the AIGaN double channel heterostructure can be calculated by self-consistently solving the one dimensional PoissonSchrödinger equation, and the electron density distribution is also extracted as shown in Fig.2. Two deep potential well are formed at the interface of AIN interlayer and $\mathrm{Al}_{0.10} \mathrm{Ga}_{0.90} \mathrm{~N}$ channel layers, corresponding to the double 2DEG channels. In addition, the lower AlGaN barrier also acts as back barrier of the upper channel, which is beneficial to promote the confinement of the 2DEG.

Fig.3 displays the high resolution $x$ ray diffraction (HRXRD) $\omega-2 \theta$ scan result of the AlGaN double channel heterostructures from symmetric (0004) reflection. The diffraction peaks of AIN nucleation layer, GaN buffer, AIGaN graded buffer, AlGaN channel and AlGaN barrier layers can be observed at $75.3^{\circ}, 71.6^{\circ}$, $72.2^{\circ}$ and $72.8^{\circ}$, indicating the distinct multi-layer structure and the sophisticated control of the growth process. Capacitance-voltage (C-V) measurement with mercury-probe configuration was performed to investigate the double channel characteristics of the heterostructures. As shown in the inset of Fig.4, two distinct capacitance steps can be observed at around $-2.5 \mathrm{~V}$ and $-10 \mathrm{~V}$ with the applied voltage swept from $0 \mathrm{~V}$ to $-15 \mathrm{~V}$, corresponding to the depletion of $2 \mathrm{DEG}$ at $\mathrm{AIN} / \mathrm{Al}_{0.10} \mathrm{Ga}_{0.90}$ interfaces. In addition, the carrier distribution properties can be extracted from $\mathrm{C}-\mathrm{V}$ curve and the result is illustrated in Fig.4. Two carrier concentration peaks locate at 23 and $78 \mathrm{~nm}$ with the values of $6.1 \times 10^{13} \mathrm{~cm}$ and $2.5 \times 10^{13} \mathrm{~cm}^{-3}$, which is in accordance with the calculated result as shown in Fig.2. Specially, no parasitic conduction channel can be observed until the test depth reached $500 \mathrm{~nm}$, suggesting the successful achievement of double channel properties of the heterostructure. In addition, the 2DEG sheet density and mobility were determined to be $1.3 \times 10^{13} \mathrm{~cm}^{-2}$ and $1130 \mathrm{~cm}^{2} / \mathrm{V} \cdot \mathrm{s}$ by the Hall effect measurement.

The standard HEMTs fabrication process was carried on the AIGaN double channel heterostructure. The device fabrication process started with ohmic contact formed with $\mathrm{Ti} / \mathrm{Al} / \mathrm{Ni} / \mathrm{Au}$ multilayer metal stack deposited by electron beam evaporation, followed by a rapid thermal anneal at $850{ }^{\circ} \mathrm{C}$ for $30 \mathrm{~s}$ in $\mathrm{N}_{2}$ atmosphere. Then, the mesa isolation was performed by $\mathrm{Cl}_{2} / \mathrm{BCl}_{3}$ inductively coupled plasma etching to a depth of $200 \mathrm{~nm}$, and 100-nm-thick SiN passivation layer was formed by plasma enhanced chemical vapor deposition. Afterwards, a gate area with a length $\left(L_{G}\right)$ of $0.8 \mu \mathrm{m}$ was defined by photolithography after etching the top $\mathrm{SiN}$ with $\mathrm{CF}_{4}$ plasma, and then a Ni/Au schottky gate electrode was deposited. The 
gate-source $\left(\mathrm{L}_{G S}\right)$ and gate-drain $\left(\mathrm{L}_{G D}\right)$ distances are 0.8 and $1 \mu \mathrm{m}$, respectively. For comparison purposes, two additional HEMTs samples based on the conventional AIGaN single channel and GaN single channel heterostructures were also fabricated, and the cross section schematics are shown in Fig.1(b) and 1(c). The device process and characteristic parameters of the additional HEMT samples are exactly the same with the AIGaN double channel HEMTs. The output and transfer properties of the devices were carried out with Keithley 4200 semiconductor parameter analyzer and the breakdown characteristics were performed using Agilent B1505A high-voltage semiconductor analyzer system.

The typical output characteristics of the HEMTs are illustrated in Fig.5, for which the $\mathrm{V}_{G S}$ and $\mathrm{V}_{\mathrm{DS}}$ were swept from $0 \sim-10 \mathrm{~V}$ and $0 \sim 10 \mathrm{~V}$. The maximum drain current density $\left(I_{\max }\right)$ and differential onresistance $\left(R_{\text {on }}\right)$ of the AlGaN single channel sample are $265.3 \mathrm{~mA} / \mathrm{mm}$ and $27.1 \Omega \cdot \mathrm{mm}$, much inferior than that of the $\mathrm{GaN}$ single channel devices. This result is in accord with the previous reports, suggesting the deficiency of AlGaN channel HEMTs in current drive capacity. However, for the AIGaN double channel HMETs, the $I_{\max }$ dramatically increases to $473 \mathrm{~mA} / \mathrm{mm}$, which is 1.8 times higher than that of AlGaN single channel HEMTs and even higher than the results of GaN single channel HEMTs. We attribute the improvement in $\mathrm{I}_{\max }$ to the superior transport properties of the AIGaN double channel heterostructures. On one hand, double channel structure possesses two potential wells along the vertical direction, and the carrier storage capability of the AIGaN conduction channel is promoted. On the other hand, although the total channel carrier density is increased, the average electron density in each channel is reduced. As a result, the carrier-carrier scattering effect is suppressed and the channel electron mobility is elevated. However, it can be observed that the $R_{\text {on }}$ of AlGaN double channel HEMTs is $12.5 \Omega \bullet \mathrm{mm}$, still larger than that of GaN single channel HEMTs. This phenomenon is related to the large contact barrier height of the AlGaN barrier layers, for which the Al composition is as high as $31 \%$. Moreover, due to the self-heating effect resulted from the high power dissipation, negative differential resistance effect can be observed for the $\mathrm{GaN}$ single channel HEMTs when $\mathrm{V}_{\mathrm{G}}>-2 \mathrm{~V}$ and $\mathrm{V}_{\mathrm{D}}>5 \mathrm{~V}$. Nevertheless, for the AIGaN channel HEMTs (both single channel and double channel), this negative differential resistance effect is significantly suppressed, manifesting the superior performance of AIGaN channel HEMTs in elevated temperature condition.

The off-state breakdown characteristics of the HEMTs based on different heterostructures are measured and shown in Fig.7. The breakdown voltage $\left(V_{b}\right)$ is defined by the criteria of leakage current reaching 0.5 $\mu \mathrm{A} / \mathrm{mm}$. It can be observed that all the three samples present hard breakdown characteristics, and the breakdown performance of AIGaN channel HEMTs are obviously better than that of the GaN channel HEMTs. The $V_{b}$ of the AIGaN double channel HEMTs is $143.5 \mathrm{~V}$, more than two times higher than that the $\mathrm{GaN}$ single channel HEMTs. Taking the $\mathrm{L}_{G D}=1 \mu \mathrm{m}$ into consideration, the $\mathrm{V}_{\mathrm{b} \text {,standard }}$ (defined by $\mathrm{V}_{\mathrm{b}} / \mathrm{L}_{\mathrm{GD}}$ ) is as high as $143.5 \mathrm{~V} / \mu \mathrm{m}$ for the AlGaN double channel HEMTs. The $\mathrm{I}_{\max }$ and $\mathrm{V}_{\mathrm{b} \text {,standard }}$ results of the AIGaN double channel HEMTs in this work are benchmarked against the GaN channel and AIGaN channel HEMTs reported by other groups in Fig.8. It is obvious that the breakdown performance of AIGaN channel HEMTs is generally better than that of GaN channel HEMTs, accompanying with the deterioration 
in $I_{\max }$. Noticeably, the $I_{\max }$ in this work is the highest result in AIGaN channel HEMTs, which is comparable to most results of the GaN channel HEMTs. Moreover, it is necessary to note that the $I_{\max }$ value in our experiments is obtained at $\mathrm{V}_{\mathrm{GS}}=0 \mathrm{~V}$, which is conservative and can be further improved by applied positive gate voltage. Therefore, these results convincingly demonstrate the enormous potential of AIGaN double channel HEMTs in microwave power device applications with requiring $1200 \mathrm{~V}$.

In summary, AlGaN double channel heterostructure is proposed to fabricate high performance HEMTs. The superior transport properties of AlGaN double channel heterostructsure is reveled, leading to the improved current drive capability of the HEMTs. In addition, the excellent breakdown performance of the AlGaN double channel HEMTs is demonstrated. The results in this work show the great potential of AlGaN double channel devices in microwave power applications in the future.

\section{Declarations}

\section{Acknowledgements}

This work is supported by the Fundamental Research Funds for the Central Universities (Grant no. JB181108), and the National Natural Science Foundation of China (Grant no. 61904139).

\section{References}

1. -Y. Liu, S.-X. Zhao, L.-Q. Zhang, H.-F. Huang, J.-S. Shi, C.-M. Zhang, H.-L. Lu, P.-F. Wang, and D. W. Zhang, Nanoscale Res. Lett. 10, 109 (2015)

2. -Y. Shih, F.-C. Chu, A. Das, C.-Y. Lee, M.-J. Chen, and R.-M. Lin, Nanoscale Res. Lett. 11, 235 (2016)

3. Ge, M. Ruzzarin, D. Chen, H. Lu, X. Yu, J. Zhou, C. D. Santi, R. Zhang, Y. Zheng, M. Meneghini, G. Meneghesso, and E. Zanoni, IEEE Electron Device Lett. 40, 379 (2019)

4. Zhang, R. Guo, S. Xu, J. Zhang, S. Zhao, H. Wang, Q. Hu, C. Zhang, and Y. Hao, Appl. Phys. Lett. 115, 072105 (2019)

5. Yang, X. Luo, T. Sun, A. Zhang, D. Ouyang, S. Deng, J. Wei, and B. Zhang, Nanoscale Res. Lett. 14, 191 (2019)

6. Nanjo, M. Takeuchi, M. Suita, T. Oishi, Y. Abe, Y. Tokuda, and Y. Aoyagi, Appl. Phys. Lett. 92, 263502 (2008)

7. Nanjo, M. Takeuchi, A. Imai, M. Suita, T. Oishi, Y. Abe, E. Yagyu, T. Kurata, Y. Tokuda, and Y. Aoyagi, Electron. Lett. 45, 1346 (2009)

8. Nanjo, A. Imai, Y. Suzuki, Y. Abe, T. Oishi, M. Suita, E. Yagyu, and Y. Tokuda, IEEE Trans. Electron Devices 60, 1046 (2013)

9. G. Baca, A. M. Armstrong, A. A. Allerman, E. A. Douglas, C. A. Sanchez, M. P. King, M. E. Coltrin, T. R. Fortune, and R. J. Kaplar, Appl. Phys. Lett. 109, 033509 (2016) 
10. Muhtadi, S. M. Hwang, A. Coleman, F. Asif, G. Simin, MVS Chandrashekhar, and A. Khan, IEEE Electron Device Lett. 38, 914 (2017)

11. Zhang, J. Cheng, M. Xiao, L. Zhang, and Y. Hao, IEEE Journal of Electron Devices Society, 6, 931 (2018)

12. G. Baca, B. A. Klein, J. R. Wendt, S. M. Lepkowski, C. D. Nordquist, A. M. Armstrong, A. A. Allerman, E. A. Douglas, and R. J. Kaplar, IEEE Electron Device Lett. 40, 17 (2019)

13. Wu, J. Zhang, S. Zhao, W. Zhang, Y. Zhang, X. Duan, J. Chen, and Yue Hao, IEEE Electron Device Lett. 40, 1724 (2019)

14. J. Freedsman, T. Hamada, M. Miyoshi, and T. Egawa, IEEE Electron Device Lett. 38, 497 (2017)

15. Chu, Y. Zhou, J. Liu, D. Wang, K. J. Chen, and K. M. Lau, IEEE Trans. Electron Devices 52, 438 (2005)

16. Wang, W. Hu, X. Chen, and Wei Lu, IEEE Trans. Electron Devices 59, 1393 (2012)

17. Zhang, Z. Wang, S. Xu, D. Chen, W. Bao, J. Zhang, J. Zhang, and Y. Hao, Appl. Phys. Lett. 111, 222107 (2017)

18. Zhang, Z. Wang, R. Guo, G. Liu, S. Xu, W. Bao, J. Zhang, and Y. Hao, Appl. Phys. Lett. 113, 233503 (2018)

\section{Figures}

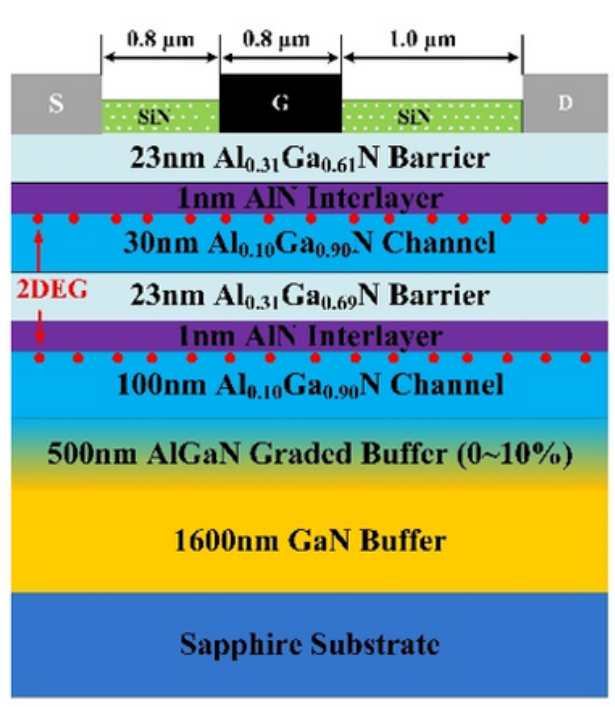

(a)

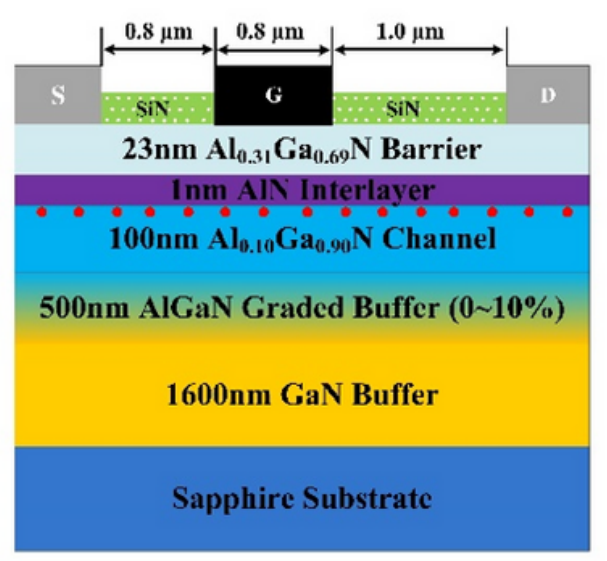

(b)

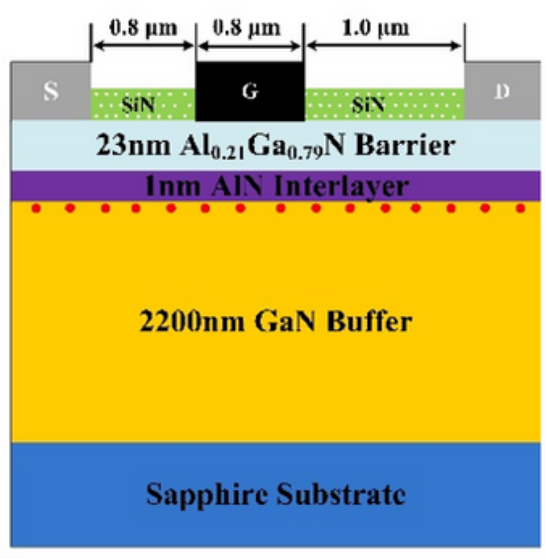

(c)

\section{Figure 1}

Cross-sectional view (not to scale) of (a) AlGaN double channel, (b) AlGaN single channel and (c) GaN single channel heterostructures (HEMTs) 


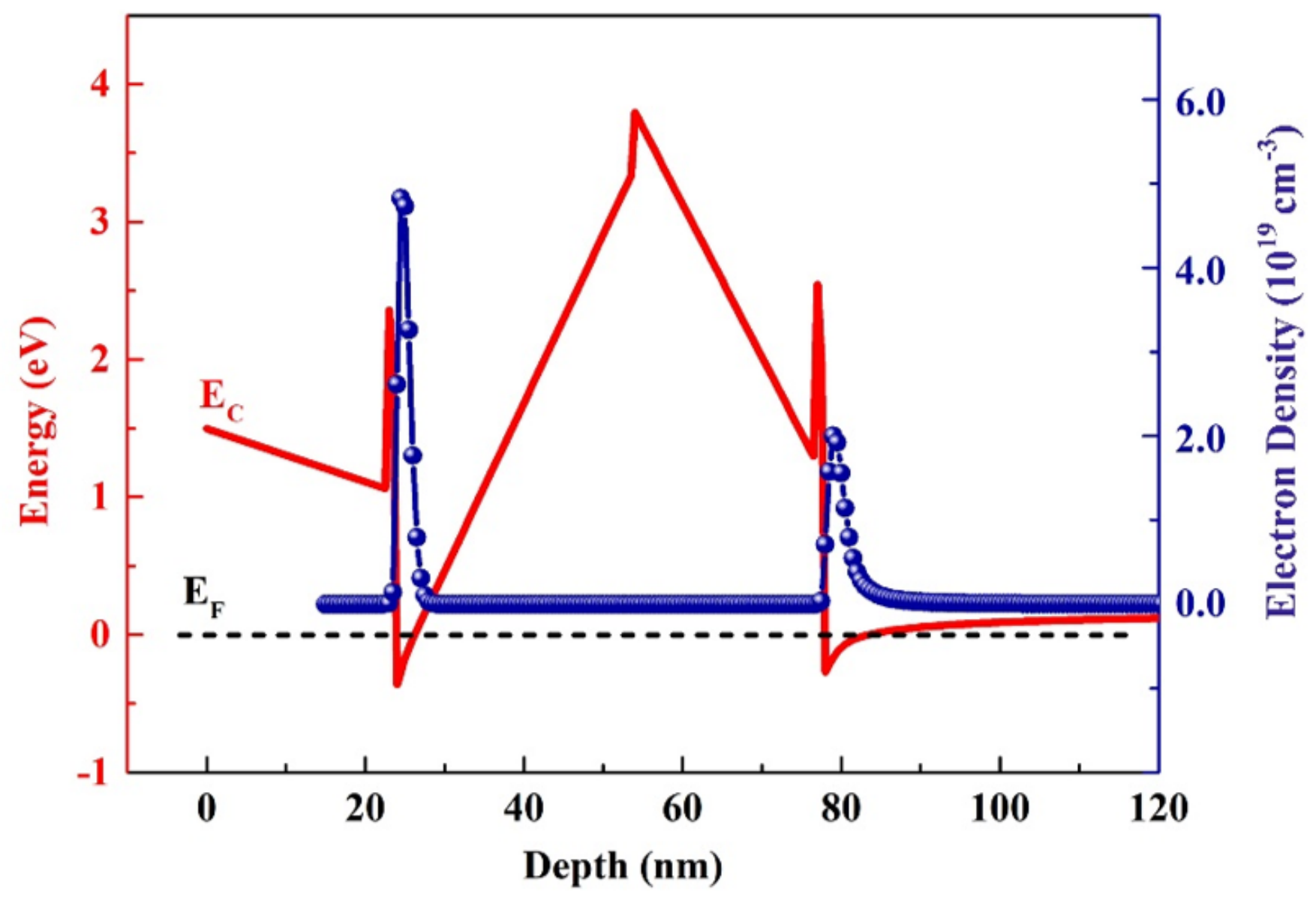

Figure 2

Conduction band diagram and electron density distribution of AIGaN double channel heterostructure 


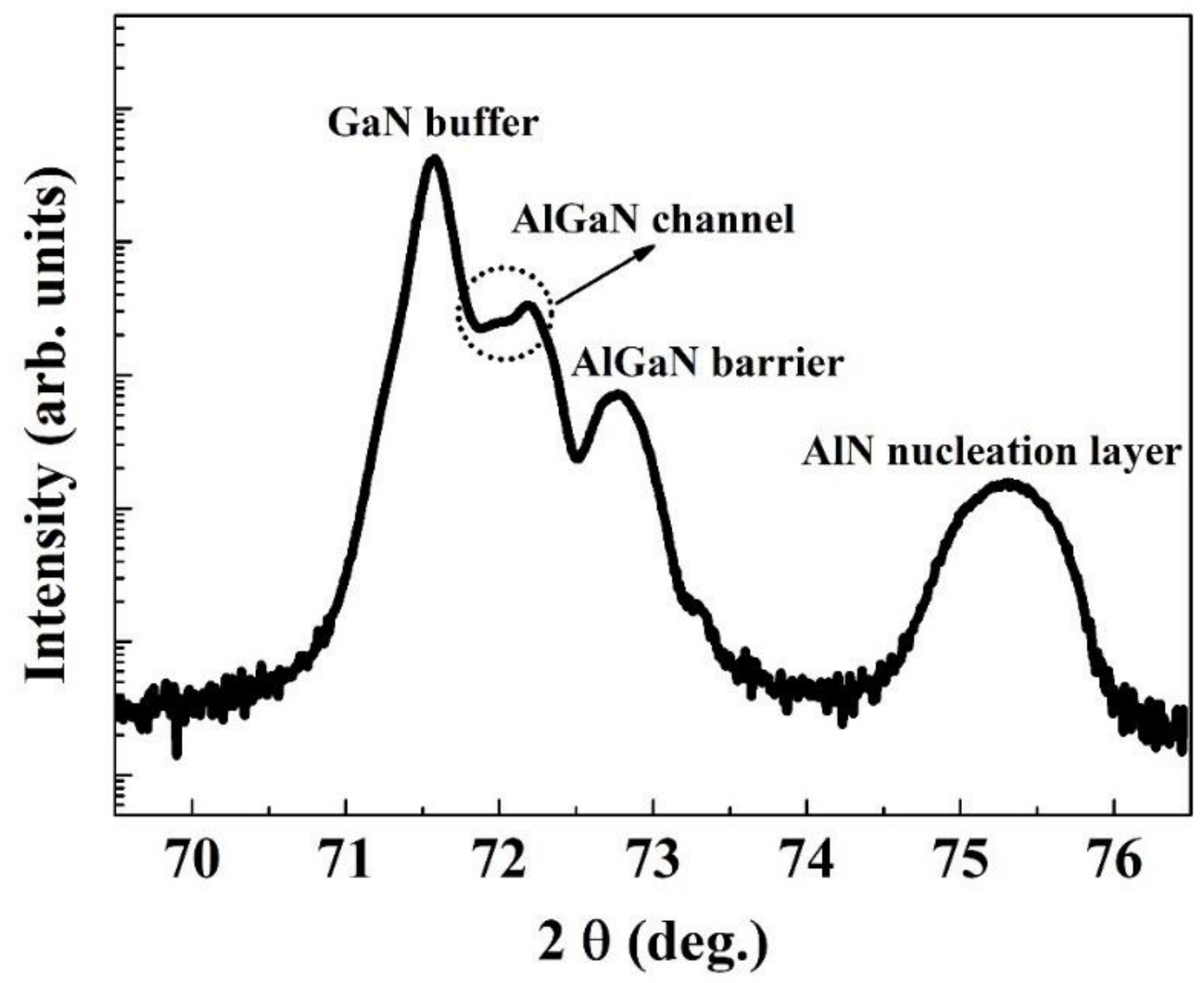

Figure 3

HRXRD (0004) plane $\omega-2 \theta$ scan of AlGaN double channel heterostructure 


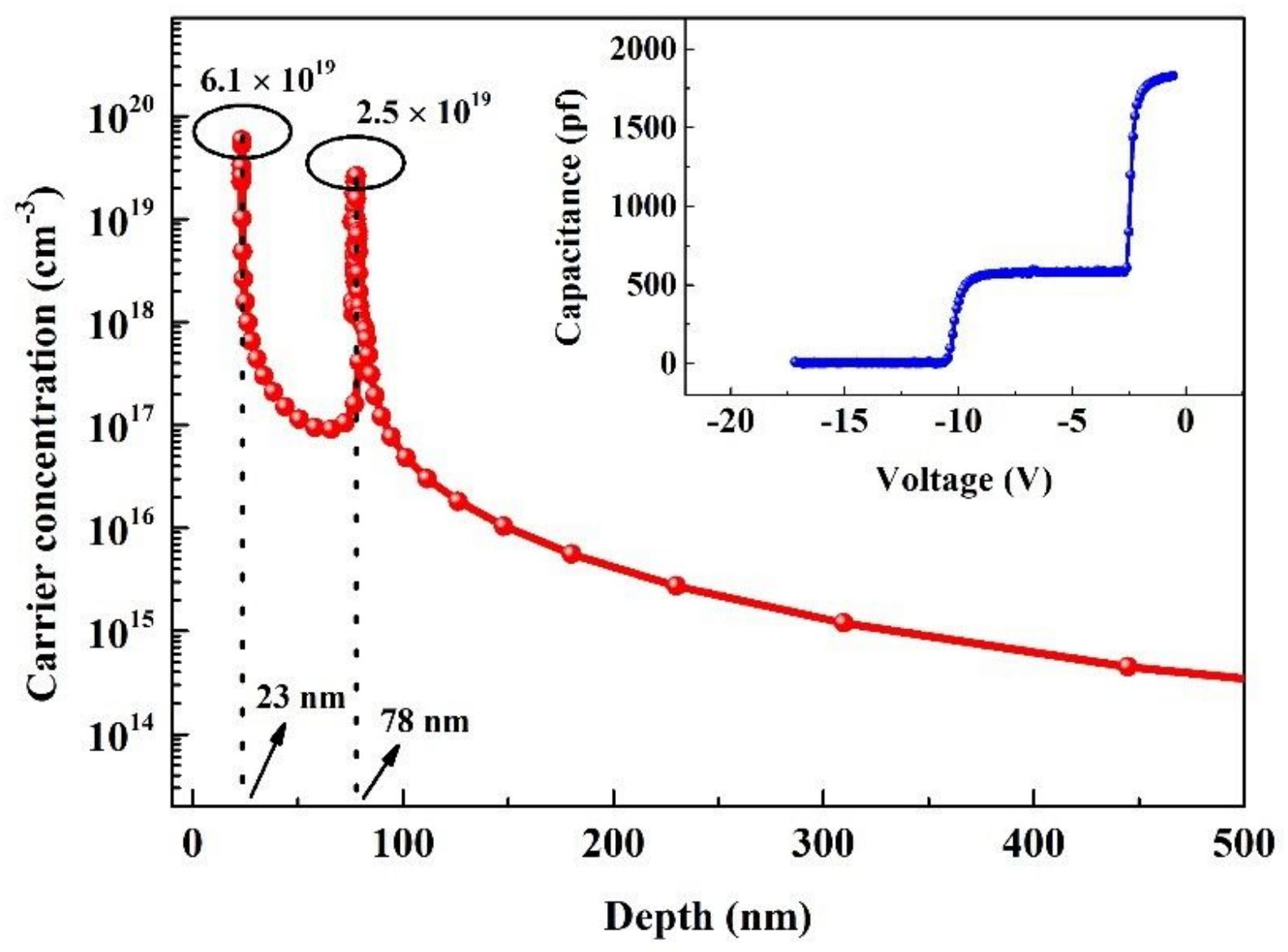

Figure 4

$\mathrm{C}-\mathrm{V}$ characteristics and electron distribution curve of AIGaN double channel heterostructure 

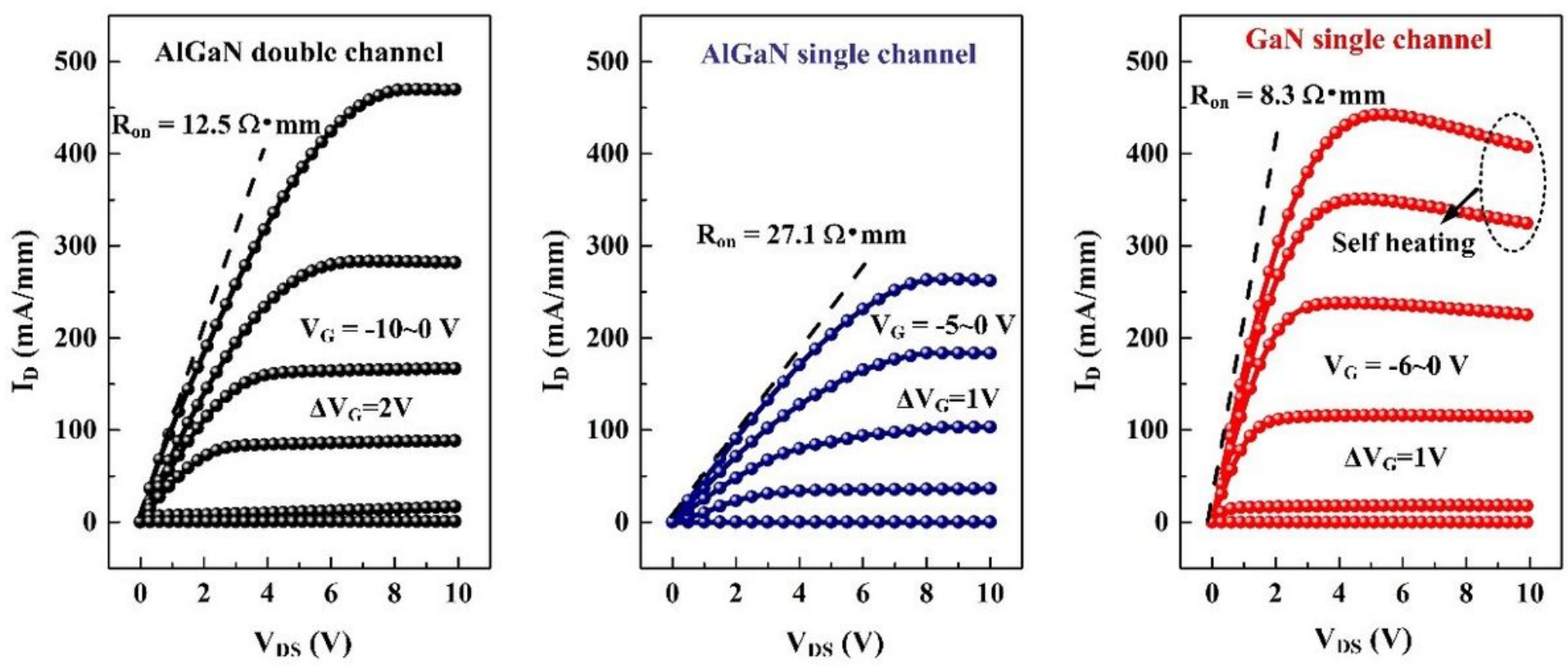

Figure 5

Output characteristics of AIGaN double channel, AlGaN single channel and GaN single channel HEMTs
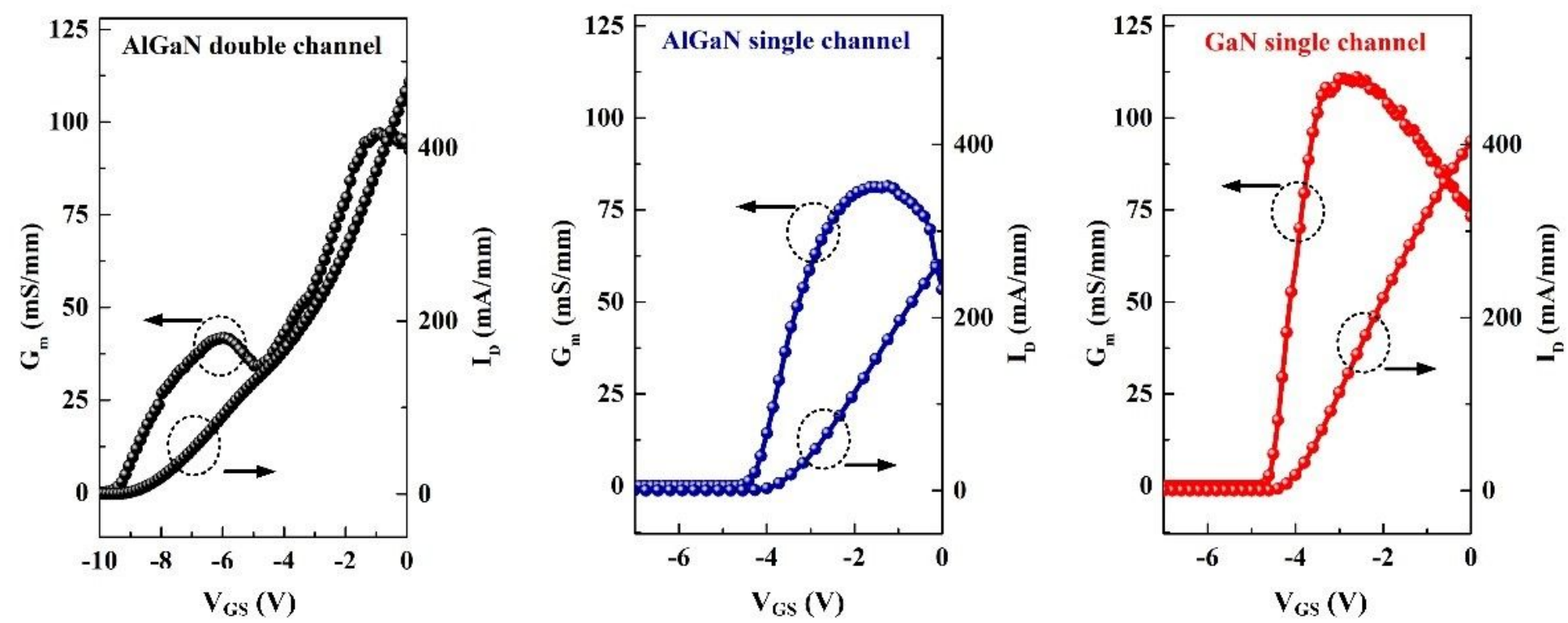

Figure 6

Transfer characteristics of AIGaN double channel, AIGaN single channel and GaN single channel HEMTs 


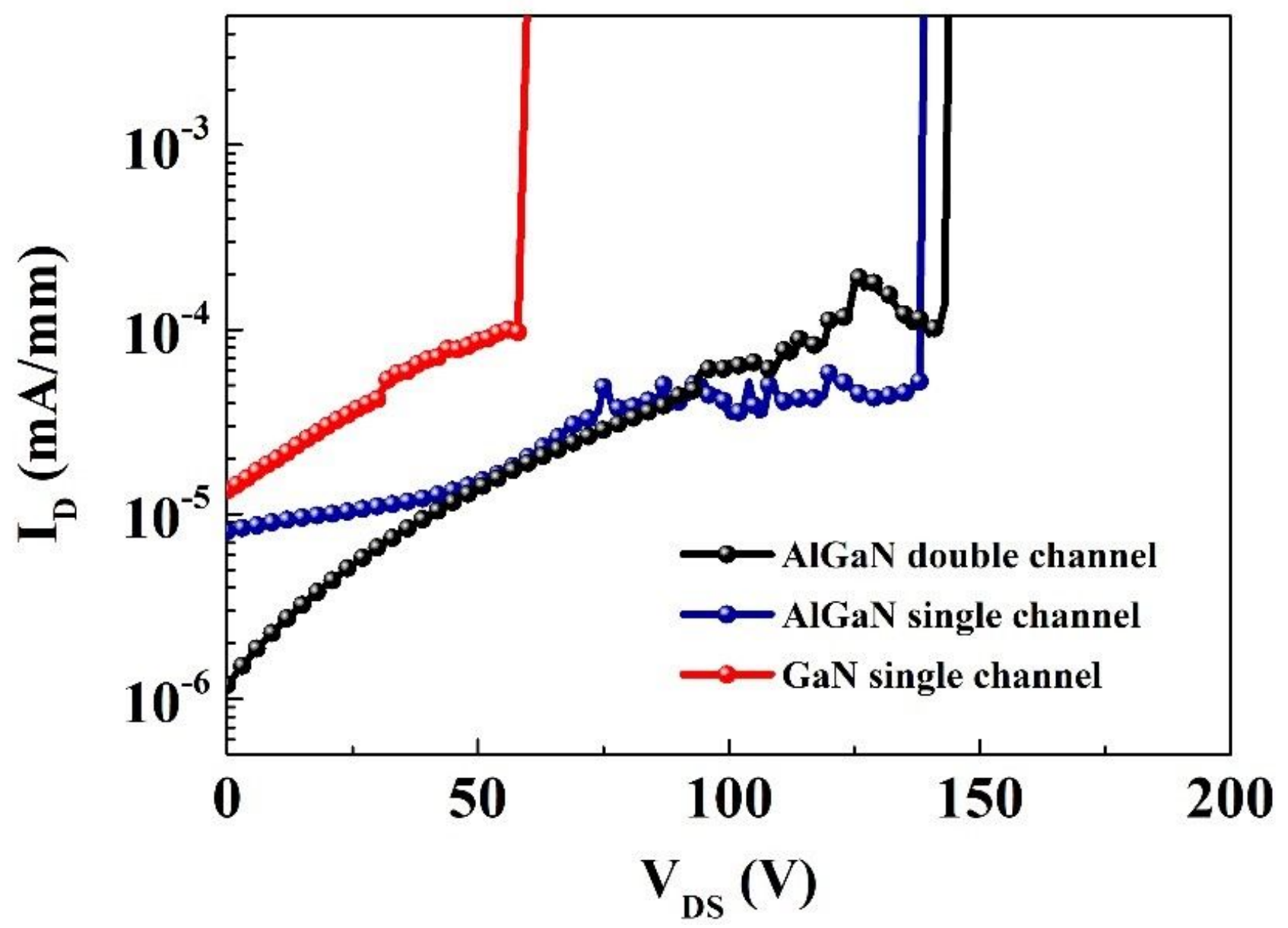

Figure 7

Breakdown characteristics of AlGaN double channel, AIGaN single channel and GaN single channel HEMTs 


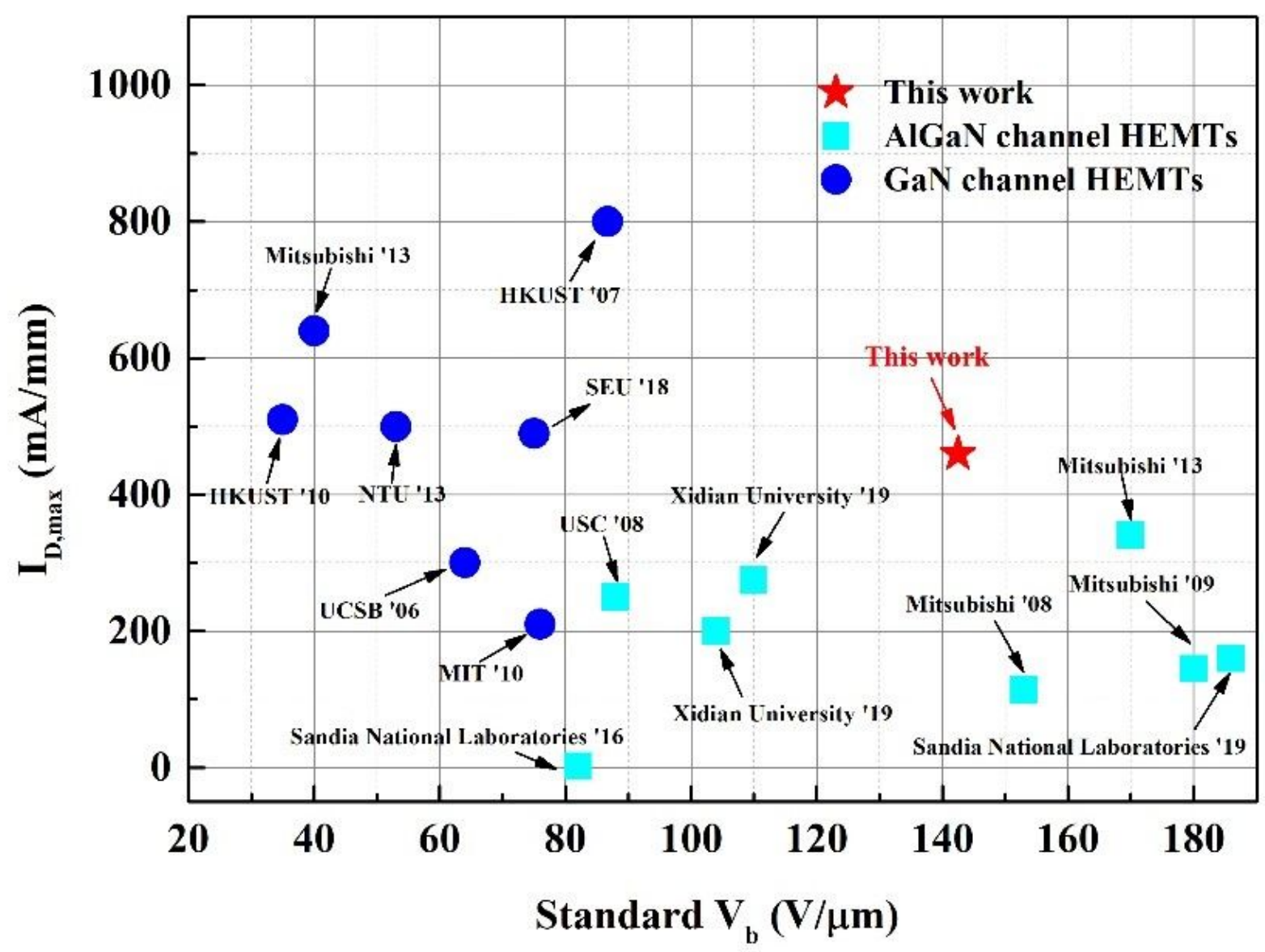

Figure 8

Benchmark of Imax and Vb,standard in state-of-the art AlGaN channel and GaN channel HEMTs 